\title{
Effect of rosiglitazone on capillary density and angiogenesis in adipose tissue of normoglycaemic humans in a randomised controlled trial
}

\author{
O. Gealekman • N. Guseva • K. Gurav • A. Gusev • \\ C. Hartigan • M. Thompson $\cdot$ S. Malkani $\cdot$ S. Corvera
}

Received: 9 April 2012 / Accepted: 8 June 2012 /Published online: 31 July 2012

(C) Springer-Verlag 2012

\begin{abstract}
Aims/hypothesis Recent reports of decreased capillary density in the adipose tissue of obese individuals suggest that an imbalance of angiogenesis and adipogenesis may, in part, underlie insulin resistance. This study aimed to determine whether the insulin-sensitising peroxisome proliferatoractivated receptor $\gamma(\operatorname{PPAR} \gamma)$ activator rosiglitazone affects adipose tissue vascularisation in normal humans.

Methods A randomised, parallel-group, investigator-blinded placebo-controlled trial was conducted with normoglycaemic volunteers with BMI $27-43$, recruited from the community at the University of Massachusetts Medical School, Worcester, MA, USA. Peri-umbilical adipose tissue biopsies were obtained before and after treatment for 6 weeks with rosiglitazone ( $8 \mathrm{mg}$ once daily) or placebo, which were randomly allocated from a sequentially numbered list. The primary outcomes were adipocyte size and capillary density measured by immunohistochemistry, and angiogenic potential assessed by capillary sprout formation in Matrigel. Secondary outcomes were serum adiponectin, glycaemic, lipid and liver function variables.

Results A total of 35 individuals fulfilling the inclusion criteria were randomised, and complete before-vs-after analyses were achieved in 30 participants (13 and 17, placebo and
\end{abstract}

Electronic supplementary material The online version of this article (doi:10.1007/s00125-012-2658-2) contains peer-reviewed but unedited supplementary material, which is available to authorised users.

O. Gealekman $\cdot$ K. Gurav $\cdot$ A. Gusev $\cdot$ S. Corvera $(\bowtie)$

Program in Molecular Medicine,

University of Massachusetts Medical School,

Worcester, MA 01615, USA

e-mail: Silvia.Corvera@umassmed.edu

N. Guseva $\cdot$ C. Hartigan $\cdot$ M. Thompson · S. Malkani

Department of Medicine,

University of Massachusetts Medical School,

Worcester, USA

rosiglitazone, respectively). Significant differences, assessed by paired two-tailed Student $t$ tests, were seen in response to rosiglitazone for adipocyte size $(3,458 \pm 202$ vs $2,693 \pm$ $\left.223 \mu^{2}, p=0.0049\right)$, capillary density $(5.6 \pm 0.5$ vs $7.5 \pm 0.5$ lumens/field, $p=0.0098)$, serum adiponectin ( $14.3 \pm 1.5$ vs $28.6 \pm 3.0 \mathrm{ng} / \mathrm{ml}, p<0.0001)$ and alkaline phosphatase (1.04 \pm 0.07 vs $0.87 \pm 0.05 \mu \mathrm{kat} / \mathrm{l}, p=0.001)$. A difference in angiogenic potential before and after treatment between the placebo and rosiglitazone groups was also seen $(-23.88 \pm 14$ vs $13.42 \pm$ $13, p=0.029$, two-tailed Mann-Whitney test).

Conclusions/interpretation Significant effects on adipose tissue vascular architecture occur after a short period of treatment with rosiglitazone in individuals with normal glucose tolerance. Improved adipose tissue vascularisation may, in part, mediate the therapeutic actions of this class of drugs.

Trial registration ClinicalTrials.gov NCT01150981

Funding The study was funded by National Institutes of Health grant DK089101 to S. Corvera, and by pilot funding from the University of Massachusetts (UMASS) Center for Clinical Translational Sciences (M. Thompson, S. Malkani and S. Corvera). Morphology core services were supported by UMASS Diabetes Endocrine Research Center (DERC) grant DK32520.

Keywords Adipose tissue expandability · Angiogenesis · Endothelial cells · Insulin resistance · Thiazolidinediones · Vascularisation

Abbreviations
ALT
AST
HOMA-IR
PPAR $\gamma$

Thiazolidinedione
Alanine transaminase

Aspartate transaminase

HOMA for insulin resistance

Peroxisome proliferator-activated receptor $\gamma$

TZD 


\section{Introduction}

Adipose tissue growth is closely associated with microvasculature development. During development, adipocytes emerge from pre-formed capillary beds, and over-nutritioninduced adipose tissue growth is coordinated with capillary expansion and with dynamic modifications of the extracellular matrix $[1,2]$. Insulin resistance in obese humans has been associated with decreased capillary density and alterations in capillary size $[2,3]$, suggesting that appropriate microcirculation is necessary for adequate adipose tissue function. Moreover, depot-dependent differences in endothelial cell function could lead to insulin resistance [3, 4] through enhanced inflammation, relative inaccessibility to nutrients and alterations in oxygen tension $[5,6]$.

Insulin resistance can be mitigated by thiazolidinediones (TZDs), which activate peroxisome proliferator-activated receptor $\gamma$ (PPAR $\gamma)$ and stimulate adipocyte differentiation. Fat depots from rodents treated with TZDs contain small adipocytes [7], which may be derived from PPAR $\gamma$ containing precursor cells embedded in the vascular wall of adipose tissue. In a previous study, we reported that treatment of obese mice with TZDs increased adipose tissue capillary density and capillary sprout formation ex vivo [8]. Stimulation of capillary growth by TZDs may contribute to mitigation of insulin resistance by improving tissue perfusion, and may possibly facilitate the formation of new adipocytes. Because these effects might constitute a new target area for therapy, we have examined whether TZDs might also affect adipose tissue vascularisation in humans. In order to discern the direct effects of TZDs from those due to changes in covariates associated with glucose intolerance (e.g. circulating glucose and insulin levels), we focused this study on individuals with normal glucose tolerance. Thus, the effects observed are likely to be attributable to the direct actions of PPAR $\gamma$ activation.

\section{Methods}

Participants Informed consent forms were signed by individuals before inclusion in the study, and protocols were approved by the University of Massachusetts Medical School Institutional Review Board. Upon enrolment, individuals were required to complete a medical history and undergo a complete physical examination, a glucose tolerance test and blood collection for measurement of fasting insulin, glucose, $\mathrm{HbA}_{1 \mathrm{c}}$, C-peptide, triacylglycerols, HDLcholesterol, LDL-cholesterol and total cholesterol, alanine transaminase (ALT), aspartate transaminase (AST), alkaline phosphatase and adiponectin. The glucose tolerance test was conducted using 75 g glucose (Trutol 75; Thermo Scientific, NERL Diagnostics LLC, East Providence, RI, USA) orally over $5 \mathrm{~min}$, with serum glucose and insulin measured at 15 , 30, 60, 90 and $120 \mathrm{~min}$.

Inclusion and exclusion criteria are shown in the electronic supplementary material (ESM). A sample of subcutaneous adipose tissue (approximately 100-200 mg wet weight) was collected from the peri-umbilical region by needle aspiration with a $14 \mathrm{~g}$ needle. Participants were then randomised to receive rosiglitazone $(8 \mathrm{mg})$ or placebo capsules (kindly provided by GlaxoSmithKline, Research Triangle Park, NC, USA), once daily for 6 weeks. Adverse event assessments were performed at weeks 2 and 4 . At the end of the study, blood and a second sample of adipose tissue (from the contralateral peri-umbilical region) were collected. The study was blinded to all enrolled individuals and participating investigators until the completion of data acquisition. A patient flow diagram prepared in accordance with CONSORT (CONsolidated Standards of Reporting Trials) is shown in ESM Fig 1.

Ex vivo angiogenesis assay of adipose tissue Briefly, freshly harvested human adipose tissue was cut into $\sim 1 \mathrm{~mm}^{3}$ pieces, which were embedded individually in wells of a 96-well plate, each containing $40 \mu \mathrm{l}$ of growth-factor-depleted Matrigel (BD Discovery Labware, Bedford, MA, USA) and cultured in EGM-2-MV (Lonza Allendale, NJ, USA) [3]. Approximately 50 independent explants per patient were embedded. In previous experiments [3] we found that $>90 \%$ of sprouting cells express endothelial cell markers, and thus represent angiogenic growth. The number of capillary branches at the periphery of the growth area was quantified by two separate observers at days 7 and 14 after embedding, and the rate of capillary outgrowth was defined as the difference in capillary branch number per explant between days 7 and 14. Measuring the growth rate decreases the variance associated with single measurements, such as failure rate (10-15\% of explants do not embed properly or fail to develop capillary branches) and the initial capillary density of the individual fragments. Data for each patient represent the average of the values obtained in all wells.

Histology of adipose tissue Adipose tissue was fixed in 4\% formaldehyde (Ted Pella, Redding CA, USA), embedded in paraffin, cut into $4 \mu \mathrm{m}$ sections and stained with rabbit antihuman von Willebrand factor (vWF) (1:200). Positive capillary lumens per area were counted by two independent observers under $\times 40$ magnification, in at least three independent sections, with at least ten fields per section for each sample. Adipocytes were identified by the single large unilocular lipid droplet, and the number of complete adipocytes per field was recorded. The mean size of adipocytes was estimated from the number of adipocytes per field and from the diameter of each adipocyte, which were recorded by at least two independent observers. 


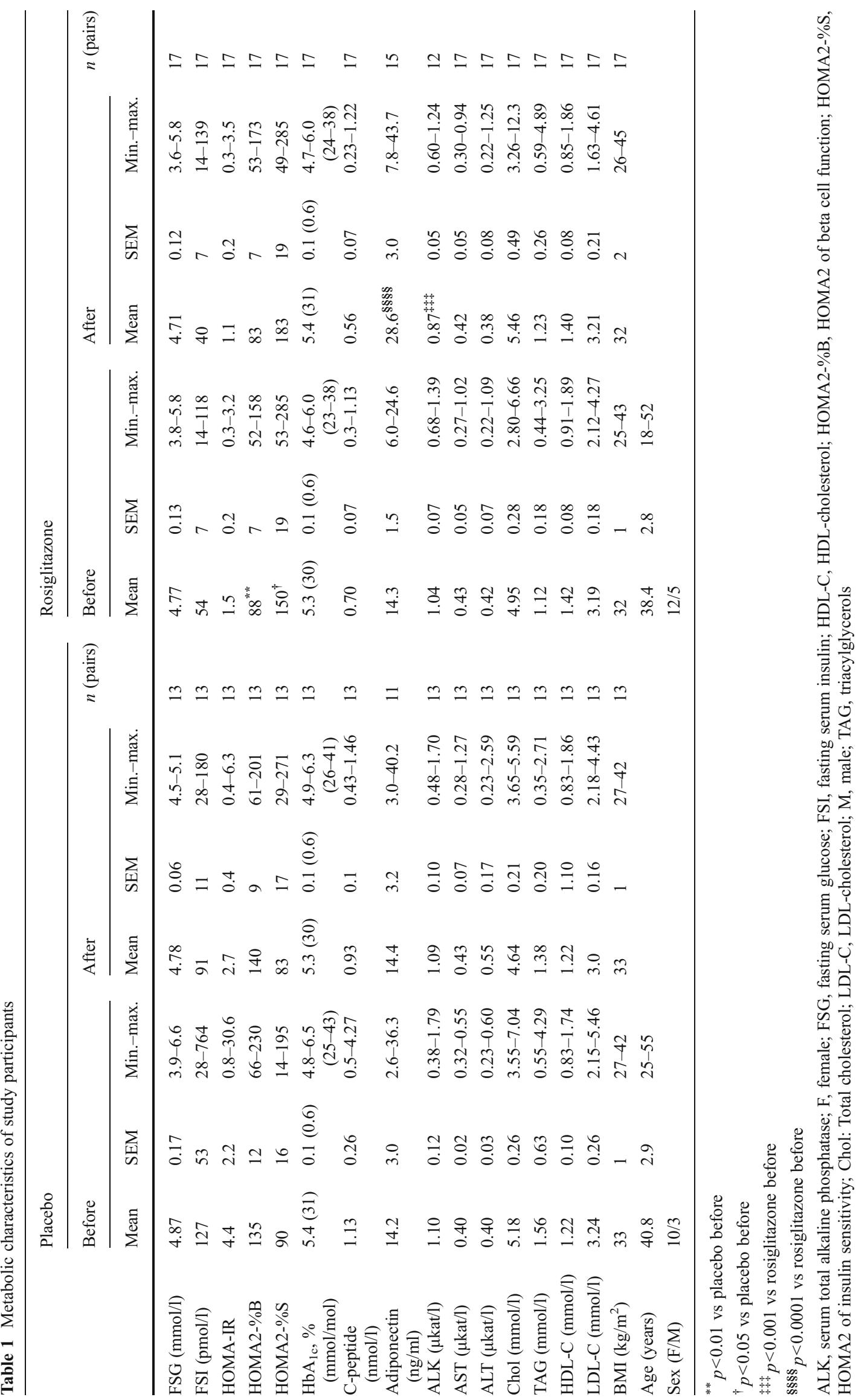


Statistical analysis Normality was assessed using the D'Agostino and Pearson Omnibus normalcy test. Two-tailed paired Student $t$ tests were used to compare values obtained before and after treatment. The two-tailed Mann-Whitney test was used to compare the difference before and after treatments between groups receiving placebo and rosiglitazone treatment. Linear regression analysis was used (GraphPad Prism).

\section{Results}

No significant before/after differences were seen in paired analyses in any variable measured in individuals exposed to placebo. In participants exposed to rosiglitazone, a highly a

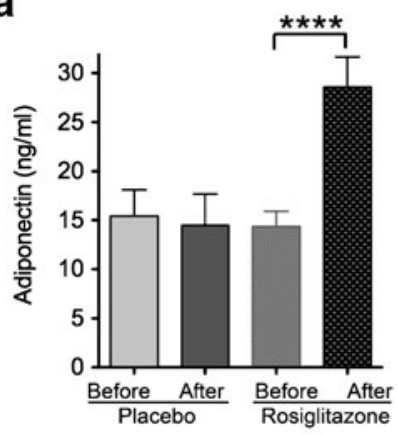

b

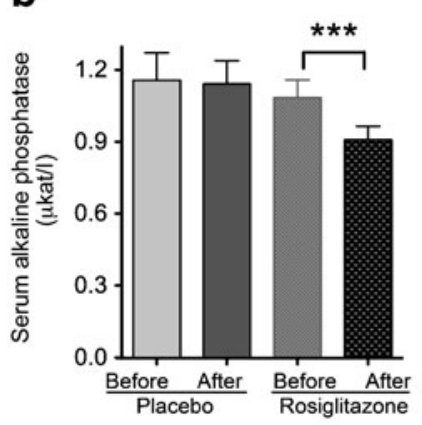

g

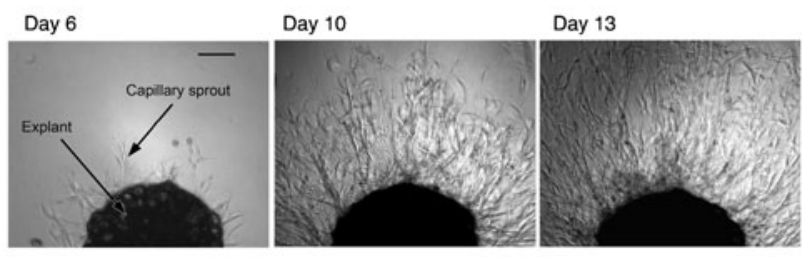

ignificant increase in serum adiponectin was observed (Table 1 and Fig. 1a, $14.3 \pm 1.5$ vs $28.6 \pm 3.0 \mathrm{ng} / \mathrm{ml}$, mean \pm SEM, before vs after rosiglitazone, respectively, $p<0.0001$, paired two-tailed Student $t$ test, 95\% CIs 11.0, 17.6 and 22.0, 35.2 , respectively, $n=15$ ). Also, a significant decrease in serum alkaline phosphatase was observed (Table 1 and Fig. $1 \mathrm{~b}, 1.04 \pm 0.07$ vs $0.87 \pm 0.05 \mu \mathrm{kat} / \mathrm{l}$, mean \pm SEM, before vs after rosiglitazone, respectively, $p=0.001$, paired two-tailed Student $t$ test, 95\% CIs 52.8, 71.6 and 44.9, 59.1, respectively, $n=12$ ). This effect, which is associated with changes in bone mineralisation, had been previously described in postmenopausal diabetic women exposed to rosiglitazone [9].

Histochemical analysis (Fig. 1c) revealed a significant increase in capillary density (Fig. 1d, $5.6 \pm 0.5$ vs $7.5 \pm 0.5$ lumens/field, mean $\pm \mathrm{SEM}$, before vs after rosiglitazone,

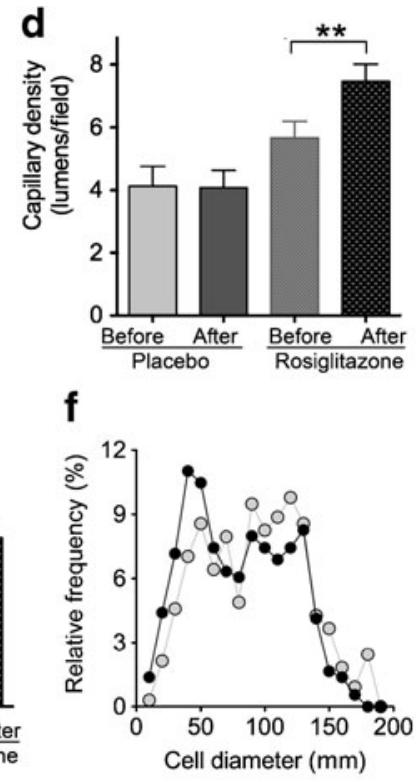

h

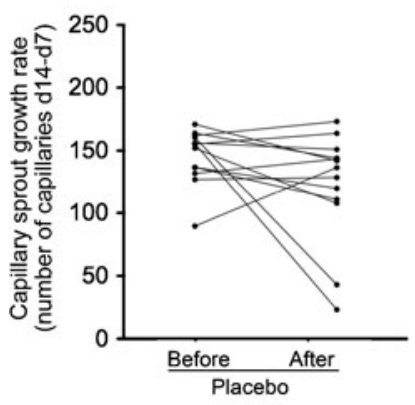

i

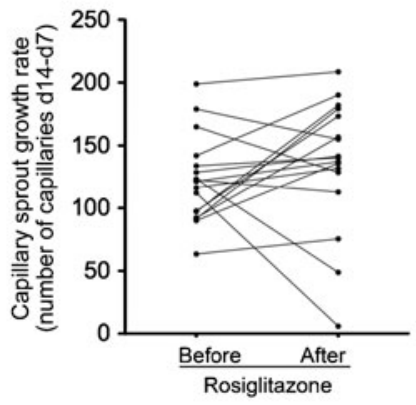

Fig. 1 Effect of rosiglitazone treatment. The plots show the mean values and SEMs for serum adiponectin (a) and serum total alkaline phosphatase (b). (c) Examples of histochemical analyses of adipose tissue; arrowheads point to capillary profiles and arrows point to small adipocytes seen in response to rosiglitazone; scale bar $100 \mu \mathrm{m}$. (d,e) Quantification of capillary density (d) and average adipocyte size (e) per field for the groups indicated on the abscissa. (f) Size distribution of adipocytes in tissue from individuals before (grey circles) and after (black circles) rosiglitazone exposure. (g) Example of capillary

C

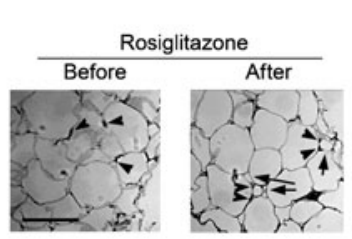

e

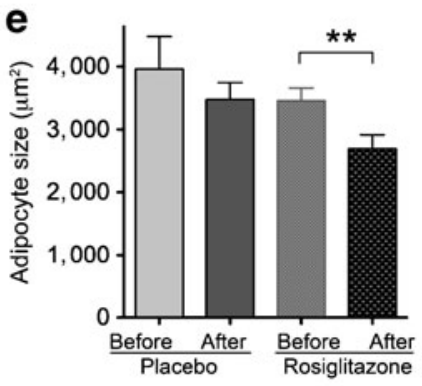

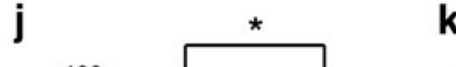
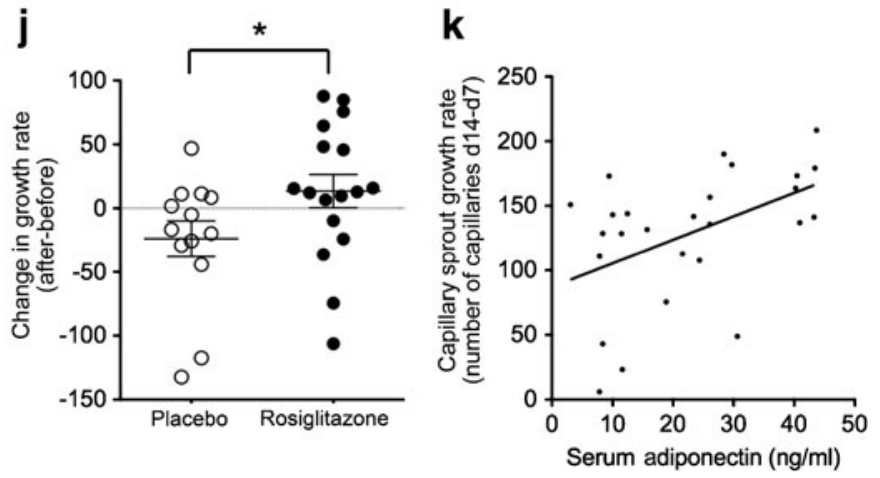

outgrowth from a single explant over time; scale bar $250 \mu \mathrm{m}$. (h,i) Before/after paired values for capillary sprout growth rate (measured as the number of capillaries on day [d] 14 less the number of capillaries on day 7) for all participants exposed to placebo (h) or rosiglitazone (i). (j) Difference between after and before growth rates for each group, with means and SEMs indicated; ${ }^{*} p<0.05 ;{ }^{* *} p<0.005 ;{ }^{* * *} p=0.001$; ${ }^{* * *} p<0.0001$. (k) Correlation between serum adiponectin levels and capillary growth rate measured at the end of the study; $p=0.0157$, $r^{2}=0.2199$ 
respectively, $p=0.0098$, paired two-tailed Student $t$ test, $95 \%$ CIs 4.4, 6.9 and 6.2, 8.8, respectively, $n=8$ ), and a decrease in mean adipocyte size (Fig. 1e, 3,458 202 vs $2,693 \pm 223 \mu \mathrm{m}^{2}$, mean \pm SEM, before vs after rosiglitazone, respectively, $p=0.0049$, paired two-tailed Student $t$ test, $95 \%$ CIs 2,980, 3,936 and 2,167, 3,220, respectively, $n=8$ ). Analysis of adipocyte size distribution revealed that this decrease correlated with the expansion of a population of small adipocytes, rather than with a decrease in overall adipocyte size (Fig. 1f).

In assays of adipose tissue angiogenesis (Fig. $1 \mathrm{~g}$ and Methods), the rate of capillary growth ex vivo was unchanged or decreased by placebo treatment (Fig. 1h), but increased after exposure to rosiglitazone (Fig. 1i). This effect was significant, as determined by the difference in the angiogenic growth rate before and after treatment observed between placebo and rosiglitazone groups (Fig. 1j, $-23.88 \pm 14$ vs $13.42 \pm 13$, mean \pm SEM, placebo vs rosiglitazone, respectively, $p=0.029$, two-tailed Mann-Whitney test, $95 \%$ CIs $-54.36,6.6$ and $-14.1,41, n=13$ pairs for placebo, 17 pairs for rosiglitazone).

Variables related to glucose tolerance, insulin sensitivity and lipid homeostasis were also measured (Table 1). An apparent non-significant difference in mean HOMA for insulin resistance (HOMA-IR) between the placebo and rosiglitazone groups is attributable to a single large fasting insulin value in the placebo group. Serum adiponectin levels at the end of the study were positively correlated with capillary growth rate (Fig. 1k). No other metabolic variable correlated with capillary growth rate in a statistically significant manner, although previous studies with larger numbers and more obese individuals have shown a negative correlation between capillary growth and insulin sensitivity [3].

\section{Discussion}

This trial in normal human volunteers demonstrates that a relatively short exposure to rosiglitazone causes significant alterations in adipose tissue architecture, increasing capillary density and the formation of small adipocytes. These changes are reflected ex vivo in an enhanced angiogenic potential, suggesting that the factors that regulate adipose tissue vasculature may be directly stimulated by PPAR $\gamma$ targeted therapies. Through changes in oxygen tension, nutrient availability or metabolite removal, an increase in capillary density may ameliorate adipose tissue insulin resistance and contribute to the therapeutic actions of these drugs.

Although this trial is limited by small sample size and participant variability, the data obtained indicate that treatment with rosiglitazone results in changes in adipose tissue capillary density, adipocyte size distribution and capillary growth ex vivo in a manner independent of changes in circulating glucose and insulin, which were not significantly altered in this study of metabolically normal individuals. Previous results have shown that isolated mature endothelial cell growth is inhibited when rosiglitazone is added directly [8]. Thus, our results are more likely to be explained by a response of other cells within adipose tissue to rosiglitazone, which indirectly affects endothelial cell growth. One possible mechanism may be the production of pro-angiogenic factors by adipocytes in the explant. One of these may be adiponectin, which has been reported to be pro-angiogenic [10] and which significantly correlated with capillary growth in this study.

Other concurrent effects of TZDs on tissue architecture, such as the modification of the extracellular matrix, may be important in regulating adipocyte expansion and function $[1,2]$. Adipose-tissue-specific angiogenesis may be a new area for target identification in the search for new therapeutics for metabolic disease.

\section{Acknowledgements}

Funding This work was funded by National Institutes of Health grant DK089101 to S. Corvera, and by pilot funding from the UMASS Center for Clinical Translational Sciences (M. Thompson, S. Malkani and S. Corvera). Morphology core services were supported by UMASS DERC grant DK32520.

Duality of interest The authors declare that there is no duality of interest associated with this manuscript.

Contribution statement $\mathrm{OG}, \mathrm{KG}$ and AG performed experiments and analysis, and critically revised the manuscript. NG, CH, MT and SM recruited and studied volunteers, acquired samples, analysed data and provided critical revisions for the manuscript. OG, NG, $\mathrm{CH}, \mathrm{MT}$, $\mathrm{SM}$ and SC designed the study, analysed data and revised the manuscript. SC, OG, MT and SM wrote the manuscript. All authors approved the final version of the manuscript.

\section{References}

1. Divoux A, Tordjman J, Lacasa D et al (2010) Fibrosis in human adipose tissue: composition, distribution, and link with lipid metabolism and fat mass loss. Diabetes 59:2817-2825

2. Spencer M, Unal R, Zhu B et al (2011) Adipose tissue extracellular matrix and vascular abnormalities in obesity and insulin resistance. J Clin Endocrinol Metab 96:E1990-E1998

3. Gealekman O, Guseva N, Hartigan C et al (2011) Depot-specific differences and insufficient subcutaneous adipose tissue angiogenesis in human obesity. Circulation 123:186-194

4. Villaret A, Galitzky J, Decaunes P et al (2010) Adipose tissue endothelial cells from obese human subjects: differences among depots in angiogenic, metabolic, and inflammatory gene expression and cellular senescence. Diabetes 59:2755-2763

5. Goossens GH, Bizzarri A, Venteclef $N$ et al (2011) Increased adipose tissue oxygen tension in obese compared with lean men is accompanied by insulin resistance, impaired adipose tissue capillarization, and inflammation. Circulation 124:67-76 
6. Pasarica M, Sereda OR, Redman LM et al (2009) Reduced adipose tissue oxygenation in human obesity: evidence for rarefaction, macrophage chemotaxis, and inflammation without an angiogenic response. Diabetes 58:718-725

7. Okuno A, Tamemoto $H$, Tobe $K$ et al (1998) Troglitazone increases the number of small adipocytes without the change of white adipose tissue mass in obese Zucker rats. J Clin Invest 101:1354-1361

8. Gealekman O, Burkart A, Chouinard M, Nicoloro SM, Straubhaar J, Corvera S (2008) Enhanced angiogenesis in obesity and in response to PPARgamma activators through adipocyte VEGF and ANGPTL4 production. Am J Physiol Endocrinol Metab 295: E1056-E1064

9. Berberoglu Z, Yazici AC, Demirag NG (2010) Effects of rosiglitazone on bone mineral density and remodelling parameters in Postmenopausal diabetic women: a 2-year follow-up study. Clin Endocrinol (Oxf) 73:305-312

10. Landskroner-Eiger S, Qian B, Muise ES et al (2009) Proangiogenic contribution of adiponectin toward mammary tumor growth in vivo. Clin Cancer Res 15:3265-3276 